\title{
ANALYSING THE IMPACT OF THE PRODUCT LIFE CYCLE ON THE IMPORTANCE OF OUTSOURCING DECISION-MAKING CRITERIA: A MANUFACTURING CASE STUDY
}

\author{
M. Cheshmberah ${ }^{1 *}$, A. Makui ${ }^{2}$, S. M. Seyedhoseini ${ }^{3} \&$ S. Yousefi $^{4}$ \\ 1,2,3 Department of Industrial Engineering \\ Iran University of Science \& Technology, Iran \\ ${ }^{1}$ mcheshmberah@iust.ac.ir; ${ }^{2}$ amakui@iust.ac.ir; ${ }^{3}$ seyedhoseini@yahoo.com \\ ${ }^{4}$ Department of Young Researchers Club, \\ Islamic Azad University, Iran \\ s.yousefie@iauctb.ac.ir
}

\begin{abstract}
This study has been carried out to discover the impact of the product life cycle (PLC) on the importance of outsourcing decision-making criteria in a manufacturing firm. The four dimensions of outsourcing decision-making were identified, based on a literature review and on the conditions of the firm: (1) core competencies, (2) information leakage risk, (3) technological capability, and (4) cost. A case study and survey research were used, along with two non-parametric tests (Friedman's test and Kendall's W). The results show in particular that the importance of 'technological capability' and 'strategic information leakage risk' does not differ across the various PLC stages. The importance of 'leakage risk of product volume information' and 'total cost' change over different stages of the product life cycle are also addressed. For the latter two criteria, the probabilities of each rating related to each criterion importance have been estimated by ordinal logistic regression, and the weights of these criteria have been calculated at each stage of the product life cycle.
\end{abstract}

\section{OPSOMMING}

Die navorsing ondersoek die invloed van produklewensiklus op die gebruik van buite leweransiers deur ' $n$ vervaardigingsonderneming. ' $n$ Besluitvormingsmetode word ontwerp en getoets deur skepping van 'n boeket van wiskundige gereedskap soos, onder andere, ordinale logistiese regressie en die logistieke funksie. Die metode word ten slotte afgerond met 'n keurige gebruik van die Wasigheidsleer.

\footnotetext{
${ }^{1}$ The author is a PhD student (Industrial Engineering) in the Department of Industrial Engineering, Iran University of Science \& Technology.

* Corresponding author
} 


\section{INTRODUCTION}

Changes in the environmental conditions of firms may lead to changes in outsourcing strategies. Consequently the outsourcing strategies of firms may change over time, altering outsourcing decisions to support their new strategies ([20], [26]). So organisations' outsourcing strategies may not remain fixed over time, and Make-or-Buy (MoB) decisions will need to be considered to support the new strategies [20]. In other words, market situation, demand fluctuation, and governmental policies force organisations to modify their outsourcing strategies ([6], [11], [32]). For instance, the relative importance of the purchasing criteria in supplier selection may change over time across different stages of PLC ([26]).

The concept of PLC has been applied in studies related to supply chain management, logistics, purchasing, etc. Birou et al et al. [4] proposed appropriate purchasing strategies proportionate to the stages of the product life cycle. Tibben-Lembke [34] considered the impact of the product life cycle on reverse logistics.

Aitken et al. [1] proposed appropriate supply chain strategies according to the stages of the product life cycle of a lighting company. Narasimhan et al. [26] proposed a mathematical model for selecting suppliers (and appropriate bid terms) according to the relative importance of multiple criteria across multiple products over their life cycles. Chang et al. [7] proposed a fuzzy multiple attribute decision-making method for supplier selection, based on different phases of the product life cycle. Wong and Ellis [38] analysed the impact of PLC on market orientation.

Hon and Xu [15] applied the concept of product life cycle to reconfiguring manufacturing systems. Chen et al. [10] proposed a nonlinear programming model for determining replenishment policies for a product life cycle by minimising the total costs of the inventory replenishment system. Ma and Yan [22] stated that in a manufacturing firm the manufacturing strategies for similar production tasks in a manufacturing firm may be stored in a knowledge base and be used to select appropriate manufacturing modes (according to specific PLC stages) for further decisions.

Che [8] proposed a decision model for developing an initiative win-win pricing strategy based on the product life cycle and a genetic algorithm. Ostlin et al. [27] studied the balance of supply and demand to provide remanufactured products during the product life cycle.

Studies in the outsourcing decision-making literature have addressed different aspects (such as a resource-based view, transactional cost theory, etc.). This problem was dealt with as a multicriteria problem ([5], [28], [23], [13], [17], [36]). The experiences of authors in various manufacturing companies raise significant questions about the impact of the product life cycle on the importance of outsourcing decision-making criteria. Moreover, the literature review shows that one of the limitations of existing research in this area is that models are static and do not address the dynamic nature of company existence and decision-making [25].

On the other hand, it is necessary to determine the relative importance of criteria. Different dimensions may be affected by importantce of of criteria such as corporate strategies and environmental conditions [20]. So in a manufacturing corporation, the importance of outsourcing decision-making criteria in a manufacturing corporation may change during product life cycle stages over time.

This paper considers the impact of the product life cycle on the importance of decisionmaking criteria related to a firm's manufacturing activities of a product, and is based on a case study and survey research methodologies. Section 2 defines each dimension of the outsourcing decision-making criteria for the development of research hypotheses and the 
research model. Section 3 explains the research methodology. Section 4 deals with the testing of the research hypotheses. In Section 5, weighting the criteria by ordinal logistic regression with respect to the TFNs is determined and discussed, and Section 6 concludes the paper.

\section{HYPOTHESES DEVELOPMENT}

The main question is whether PLC has any impact on the importance of the criteria for outsourcing decision-making, and how the importance of a criterion might be altered over time through the stages of the product life cycle. Other factors, such as market conditions, demand fluctuations, and governmental policies ([6], [11], [32], [20]) are also considered. By adopting Porter's value chain, the range of processes are manufacturing processes (or activities) related to a specific product that is named 'operations' [29].

\subsection{The dimensions of outsourcing decision-making}

Based on the literature reviewed and on the conditions of the studied firm, four dimensions affecting outsourcing decision-making are identified, as shown in Figure 1. These are:

- Core competency dimension

- Information security (leakage risk) diemnsion

- Technological dimension

- Economic dimension

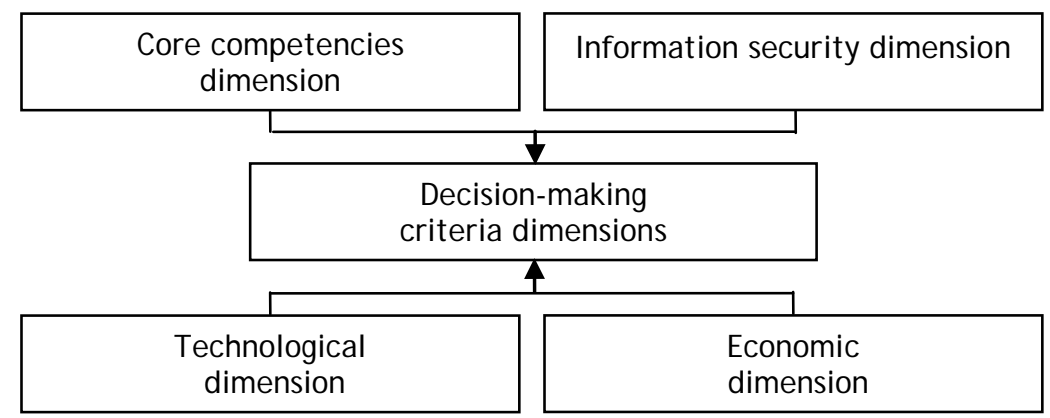

Figure 1: Dimensions that influence outsourcing decision-making in the studied firm

\subsubsection{Core competencies dimension}

Prahalad and Hamel [30] argued that core competencies in a company have at least three attributes: they

- provide a potential approach to various markets

- $\quad$ play an important role in customer intuition; and

- $\quad$ are difficult to imitate (by competitors).

All goods and services that are considered to be core competencies should be retained and performed internally (insourcing); and in these cases, outsourcing should necessarily be avoided by firms ([35], [24], [2]).

\subsubsection{Information security (leakage risk) dimension}

One important issue in outsourcing decision-making is how information security can be realized ([19]). The risks of information leakage can be divided into two categories for the studied firm:

- Risk of strategic information leakage, and

- risk of volume product information leakage 
Information on sales, strategic directions and policies, target (niche) markets, etc. are examples of confidential strategic information. Breach of confidentiality may result in competitive damage [14] and irreparable losses to organisations.

The second category of information leakage is volume information about products. Knowing the exact quantity of a product may be important for competitors. Outsourcing an activity of a product may disclose such information. If this information is important and the impact of related risk is high, it is important to protect it as much as possible. Related risks concerning that activity should also be managed. Performing the neccesary activity internally or externally is significant. To conclude, it is questionable whether the importance of information leakage risks (strategic and product) at different stages of the product life cycle will be changed.

\subsubsection{Technological dimension}

The technological capability to perform processes or activities both about the firm and about the potential suppliers is the third dimension of outsourcing decision makingdecisionmaking. Many authors mention 'technological capability' as a major factor for outsourcing decision-making ([5], [11], [13], [17], [18], [37], [31], [28], [36]).

Technological capabilities generally follow the life cycle and maturity level as well as the position of competitors [37]. Obviously, a specific level of technological capability is needed to meet the minimum requirements for product quality. However, the supplier with higher levels of technological capability is preferred [12]. It is necessary to know whether the importance of technological capability varies across different stages of PLC.

\subsubsection{Economic dimension}

The fourth outsourcing decision-making dimension of the study is the economic dimension. 'Total cost' and 'cost saving' have been used as major criteria for the economic dimension of outsourcing decision-making in many works ([2], [5], [13], [17], [18], [28], [20], [36]). In this study, 'total cost' is selected as the economic criterion and how it may change as process (activity) changes across PLC stages.

\subsection{Hypotheses}

According to the outsourcing decision-making criteria for the studied firm, the research model was established. The attendant hypotheses are expressed as follows (see Figure 2):

Ha: The importance of technological capability differs at various stages of the PLC.

$\mathrm{Hb}$ : The importance of strategic information leakage risk differs at various stages of the PLC.

Hc: The importance of leakage risk of product volume information differs at various stages of the PLC.

Hd: The importance of total cost differs at various stages of the PLC.

\section{RESEARCH METHODOLOGY}

Case study and survey research approaches were applied in this study. The studied organisation is an Iranian manufacturing firm in the field of aviation. A cross-functional team was formed to analyse the research goal and data collection. Almost 40 experts participated in the team, from production, planning, quality assurance and control, research and development, procurement, sales and CRM departments. All team members were related to outsourcing affairs or decision makingdecision-making processes while performing normal functions. 
Does the criterion importance differ at various stages of PLC?

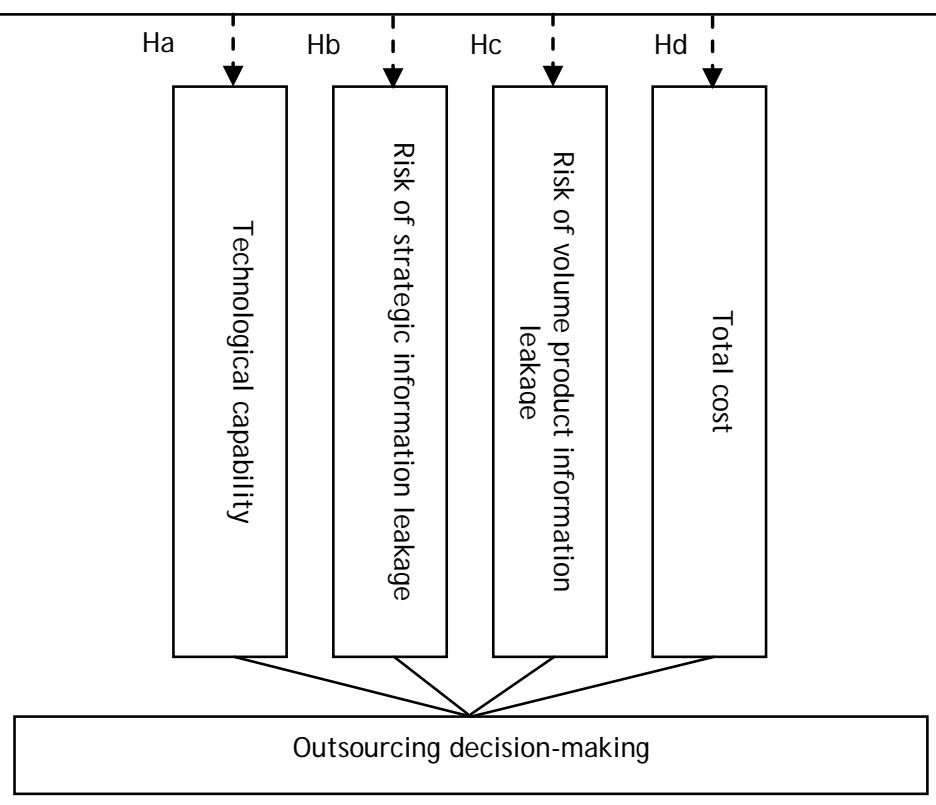

Figure 2: Research model

The team participated in lectures (description of research goal) and workshops (data gathering). A questionnaire was distributed among them as a survey instrument. The questionnaire was developed specifically to measure the importance of each criterion at different stages of PLC, and included items using a Likert scale (ranking from $1=$ very low to $5=$ very high). To finalise and ensure the instrument's validity, the questionnaire was assessed and tentatively completed by a number of manufacturing experts (staff at the studied firm) and an academic consultant before data gathering. Lastly, data were collected by distributing the questionnaire to the experts who participated in the workshops. In the study, Cronbach's alpha is 0.711 , which indicates an acceptable internal consistency of items in the survey instrument [33].

\section{RESULTS OF FRIEDMAN'S TEST}

To examine whether significant differences exist in the criteria importance at various stages of the PLC, two non-parametric tests (Friedman's test and Kendall's W) were used. In Friedman's test, the null hypothesis is that there is no real difference between the $n$ objects (stages). Kendall's coefficient of concordance (W) is a measure of the agreement among several judges who are assessing a set of objects [21]. Kendall's $W$ is a value between 0 and 1 , where 0 indicates no agreement and 1 indicates complete agreement. The results from both the Friedman's test and Kendall's W coefficient of concordance are shown in Table 1 . This study tested the hypotheses for statistical significance using the 5 percent significance level $(\alpha=0.05)$.

\begin{tabular}{lcccc}
\hline & Ha & Hb & Hc & Hd \\
\hline Friedman's Test p-value & .066 & .096 & .000 & .000 \\
Kendall's W Coefficient of Concordance & 0.086 & 0.056 & 0.804 & 0.652 \\
Support & No & No & Yes & Yes \\
\hline
\end{tabular}

Table 1: Results of Friedman's Test and Kendall's W Coefficient of Concordance

Using Friedman's test to compare the medians across all four life cycle stages confirms that no significant differences were observed in 'importance of technological capability' and 
'importance of strategic information leakage risk' across PLC stages, because $\mathrm{Ha}$ and $\mathrm{Hb}$ are not supported ( $p$-value $>0.05$ ).

Table 1 also reveals that the Friedman's Test $\mathrm{p}$-value for $\mathrm{Hc}$ and $\mathrm{Hd}$ is 0.00 ; so data relevant for testing $\mathrm{Hc}$ and $\mathrm{Hd}$ strongly support $\mathrm{Hc}$ and $\mathrm{Hd}(\mathrm{p}$-value $<0.05)$. This means that the importance of 'risk of product volume information leakage' and 'total cost' differs across various PLC stages.

The results of Table 1 also show that the total rankings between 'importance of leakage risk of product volume information' and 'stages of $\mathrm{PLC}$ ' have extremely strong agreement ( $\mathrm{p}$-value $\varangle$.05; Kendall's $\mathrm{W}=0.804$ ); as well as the total rankings between 'importance of total cost' and 'PLC stages' ( $p$-value $\varangle 0.05$; Kendall's $W=0.652$ ) which are in strong agreement. This means that the importance of 'leakage risk of product volume information' and 'total cost' are different across various PLC stages, and the importance of 'strategic information leakage risk' and of 'technological capability' is independent of PLC stages.

\section{WEIGHTING THE CRITERIA BY ORDINAL LOGISTIC REGRESSION}

The findings indicate that the decision-makers of the studied firm have to adjust the weighting criteria by considering the PLC. For the criteria that have been shown to depend on the various stages of product life cycle, it is possible to use ordinal logistic regression to determine the weights at each stage of the product life cycle. If $\mathrm{x}$ is considered as an independent variable, and $Y$ as a dependent (response) variable, the regression model seeks an estimate of $E(Y \mid X)$. If $E(Y \mid X)$ is represented by $\Pi(x)$, then by using the logistic regression model, $\Pi(\mathrm{X})$ can be considered as in the following equation ([16]):

$\boldsymbol{\pi}(\mathbf{x})=\frac{e^{\beta_{0}+\beta_{1} x}}{1+e^{\beta_{0}+\beta_{1} x}}$

Equation (1.1) can be reorganised as a logit transformation form (Equation 1.2):

$$
\ln \left[\frac{\pi(\mathrm{x})}{1-\pi(\mathrm{x})}\right]=\beta_{0}+\beta_{1} \cdot \mathrm{x}
$$

One may now consider determining the relation between the importance of each criterion as an output (response) variable and the product life cycle as an input or independent variable. The independent variable is an ordinal variable with four stages: introduction, growth, maturity, and decline. The output (dependent) variable is an ordinal variable with five rating scales: very low, low, medium, high, and very high. In the ordinal logistic regression, an 'event' is defined as the occurrence of each output variable or less than them; so the following equation may be defined:

$$
\theta_{\mathrm{j}}=\frac{\mathrm{p}(\text { score } \leq \mathrm{j})}{\mathrm{p}(\text { score }>\mathrm{j})}
$$

Equation (1.3) can be rewritten in another form as equation (1.4):

$$
\boldsymbol{\theta}_{\mathrm{j}}=\frac{\mathbf{p}(\text { score } \leq \mathbf{j})}{\mathbf{1}-\mathbf{p}(\text { score } \leq \mathbf{j})}
$$

According to the logic equation (1.2), it is possible to set up equation (1.5) for an independent variable $(x)$ in an ordinal logistic regression model as follows:

$$
\ln \theta_{\mathrm{j}}=\alpha_{\mathrm{j}}-\boldsymbol{\beta} \cdot \mathrm{x}
$$


by combination of equation (1.4) and equation (1.5), which produces equation (1.6).

$=\frac{1}{1+\mathrm{e}^{-\left(\alpha_{j}-\beta . x\right)}}$

The events in an ordinal logistic model are cumulative scores, not individual scores but cumulative scores. Figures 3 and 4 are the cumulative percentage plots of the ratings, with separate curves for various stages of the product life cycle. The figures demonstrate that the ordinal regression model fits a function of the four curves.

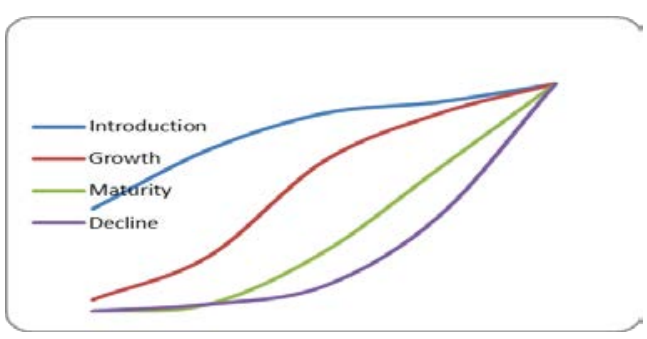

Figure 3: Cumulative percentage plot of the importance of total cost at various stages of the PLC

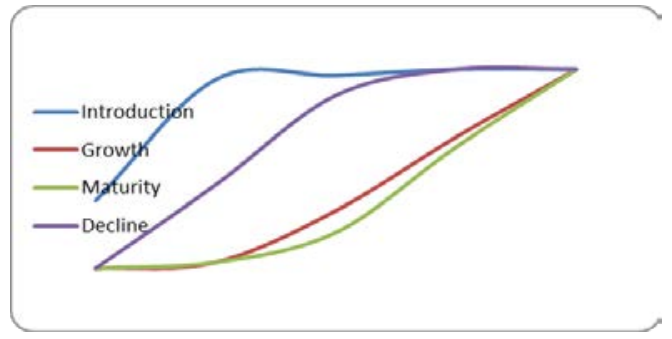

Figure 4: Cumulative percentage plot of the importance of leakage risk of product volume information at PLC stages

Table 2 shows the coefficients of the ordinal logistic regression model for the dependent variable (the importance of total cost) and the independent variable (PLC) as calculated by the Statistical Package for Social Sciences software (SPSS).

\begin{tabular}{|c|c|c|c|c|c|}
\hline & & Estimate & Std. Error & df & Sig. \\
\hline \multirow[t]{4}{*}{ Threshold } & {$[$ Scor. Cs = 1] } & -5.203 & .544 & 1 & .000 \\
\hline & [Scor.Cs = 2] & -3.746 & .471 & 1 & .000 \\
\hline & [Scor.Cs = 3] & -1.972 & .382 & 1 & .000 \\
\hline & {$[$ Scor.Cs $=4]$} & -.348 & .323 & 1 & .280 \\
\hline \multirow[t]{4}{*}{ Location } & [PLC. Stages $=1]$ & -5.089 & .585 & 1 & .000 \\
\hline & [PLC. Stages=2] & -2.822 & .492 & 1 & .000 \\
\hline & [PLC. Stages=3] & -.883 & .441 & 1 & .045 \\
\hline & [PLC. Stages $=4]$ & $0^{\mathrm{a}}$ & . & 0 & \\
\hline
\end{tabular}

Table 2: Estimated coefficients of ordinal logistic regression model for dependent variable (the importance of total cost) and the independent variable (PLC) 
The coefficients in Table 3 may be used in the logistic regression model to estimate the cumulative probabilities for each event, and for each rating of the dependent variable.

\begin{tabular}{|c|c|c|c|c|}
\hline Model & -2 Log Likelihood & Chi-Square & df & Sig. \\
\hline Null Hypothesis & 47.661 & & & \\
General & 42.814 & 4.847 & 9 & .847 \\
\hline
\end{tabular}

Table 3: Test of parallel Lines

In fitting the ordinal regression, it was assumed that the relationships between the independent variables and the logits are the same for all logits. If the lines or planes are parallel - as in the SPSS results of Table 3 - the observed significance level for the change should be large, since one would not wish to reject the null hypothesis that the lines are parallel (see Table 4).

As an example, by using equation (1.6), the cumulative probabilities for each rating scale of the dependent variable (the importance of total cost) with respect to the introduction stage for the product are calculated. By considering the estimated coefficients of the ordinal logistic regression model shown in Table 2, the cumulative probabilities for each rating scale are:

Prob $($ Score $=1 /$ PLCS $=$ Introduction $)=1 /\left(1+e^{5.203-5.099}\right)=0.47153$

Prob (Score $<=2 /$ PLCS $=$ Introduction $)=1 /\left(1+e^{\text {a.746-5.089 }}\right)=0.79298$

Prob (Score $<=3 /$ PLCS $=$ Introduction $)=1 /\left(1+e^{1.972-5.089}\right)=0.95759$

Prob (Score $<=4 /$ PLCS $=$ Introduction $)=1 /\left(1+e^{0.348-5.089}\right)=0.99135$

Prob (Score $<=5 /$ PLCS $=$ Introduction $)=1$

According to the discreteness of the response variable, the probabilities of each rating scale can be estimated (equation 1.7).

$$
(\text { score }=\mathbf{j})=\mathbf{p}(\text { score } \leq \mathbf{j})-\mathbf{p}(\text { score } \leq \mathbf{j}-\mathbf{1})
$$

For the importance of total cost in the introduction stage, the probabilities are:

Prob (Score $=1 /$ PLCS $=$ Introduction $)=0.47153$

Prob $($ Score $=2 /$ PLCS $=$ Introduction $)=\operatorname{prob}($ score $<=2)-\operatorname{prob}($ score $<=1)=0.32145$

Prob $($ Score $=3 /$ PLCS $=$ Introduction $)=\operatorname{prob}($ score $<=3)-\operatorname{prob}($ score $<=2)=0.16461$

Prob $($ Score $=4 /$ PLCS $=$ Introduction $)=\operatorname{prob}($ score $<=4)-\operatorname{prob}($ score $<=3)=0.03376$

Prob $($ Score $=5 /$ PLCS $=$ Introduction $)=1-\operatorname{prob}($ score $<=4)=0.00865$

Similarly, the probabilities of the various ratings of the importance of the total cost criterion for other stages may be estimated. In the same way, by defining the regression model for the importance of leakage risk of product volume information, the probabilities for each rating scale (very low, low, medium, high, and very high) for each stage of the product life cycle may be estimated.

Since the rating scales are linguistic variables (very low, low, medium, high and very high), one may use a fuzzy logic approach to decrease the vagueness of such variables.

A fuzzy linguistic variable reflects different aspects of the human language. When the values or meanings of a linguistic factor are being reflected, the resulting variable must also reflect appropriate modes of change for that linguistic factor. Moreover, variables describing a human word or sentence may be divided into numerous linguistic criteria, such as equally important, moderately important, strongly important, very strongly important, and extremely important ([9]). This paper uses a triangular fuzzy membership function 
(Figure 4) and triangular fuzzy numbers (Table 4) as the most applicable among other fuzzy methods to calculate the weights of decision-making criteria effectively.

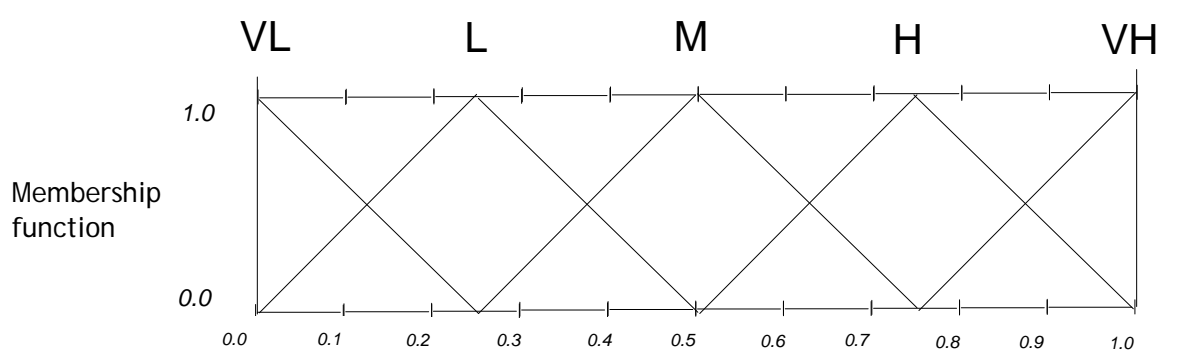

Figure 4: Triangular fuzzy membership function for five scores

\begin{tabular}{|c|c|}
\hline Linguistic terms & Linguistic values \\
\hline Very high & $(0.75,1.0,1.0)$ \\
\hline High & $(0.5,0.75,1.0)$ \\
\hline medium & $(0.25,0.5,0.75)$ \\
\hline low & $(0,0.25,0.5)$ \\
\hline Very low & $(0,0,0.25)$ \\
\hline
\end{tabular}

Table 4: Linguistic scales for the importance of criteria

The basic arithmetic rules for TFNs like $(a, b, c)$ and $(d, e, f)$ are as follows ([39]):

Addition:

$[a, b, c]+[d, e, f]=[a+d, b+e, c+f]$

Subtraction:

Scalar multiplication:

$[a, b, c]-[d, e, f]=[a-d, b-e, c-f]$

$$
k \times[a, b, c]=[k a, k b, k c] k>0
$$

To convert the importance of each criterion to a certain weight in order to use them for the decision-making process with respect to the arithmetic rules of TFNs, one applies equation (1.11). The weights of each criterion are determined, however, with respect to the product life cycle:

$$
\mathbf{W}_{\mathrm{ij}}=\sum_{\mathrm{k}} \mathbf{P}_{\mathrm{ij}}\left(\mathbf{r}_{\mathrm{k}}\right) \cdot \widetilde{\mathbf{r}}_{\mathrm{k}}
$$

where:

$\mathbf{W}_{\mathrm{ij}}$ : Weight of criterion i at stage j;

$P_{i j}\left(r_{k}\right)$ : Probability of rating $k$ on importance criterion $i$ at stage $j$;

$\widetilde{\mathbf{r}_{\mathrm{k}}}$ : Rating $\mathrm{k}$ in triangular fuzzy number form

$\mathrm{i}=1,2,3, \ldots ; j=\mathrm{l}, \mathrm{G}, \mathrm{M}, \mathrm{D} ; \mathrm{k}=$ 'very low', low, medium, high, 'very high'

As an example, the weights of the total cost in the introduction stage may be determined. In the case of 'technological capability' and 'strategic information leakage risk' with respect to the independency of the product life cycle, median values will be considered for calculations (the medians are very high in all stages of the PLC).

For the other two criteria (leakage risk of product volume information, and total cost) where their importance changes over the four stages of the product life cycle, equation (1.11) will be used to estimate the weights of the criteria. Calculating the weight of the total cost in the introduction stage of the PLC performed as follows: 
Weight $($ Total cost $/$ PLCS $=$ Introduction $)=0.47153 *(0.0,0.0,0.25)+0.32145 *(0.0$, $0.25,0.5)+0.16461 *(0.25,0.5,0.75)+0.03376 *(0.5,0.75,1.0)+$ $0.00865 *(0.75,1.0,1.0)=(0.0625,0.1925,0.4375)$

The weights of the four criteria based on triangular fuzzy numbers are given in Table 5 . It is now possible to use the estimated weights with every multi-criteria decision-making approach to determine the preferred alternative(s) and make decisions for the insourcing or outsourcing of manufacturing activities with respect to product life cycle considerations.

\begin{tabular}{|c|c|c|}
\hline Criteria & Weighting origin & Weight (TFN) \\
\hline Importance of technological capability & Median of judgements & $(0.75,1.0,1.0)$ \\
\hline $\begin{array}{c}\text { Importance of risk of strategic information } \\
\text { leakage }\end{array}$ & Median of judgements & $(0.75,1.0,1.0)$ \\
\hline $\begin{array}{c}\text { Importance of leakage risk of product volume } \\
\text { information }\end{array}$ & Equation $(1.11)$ & $(0.0075,0.1725,0.42)$ \\
\hline Importance of total cost & Equation $(1.11)$ & $(0.0625,0.1925,0.4375)$ \\
\hline
\end{tabular}

Table 5: The weights of criteria in triangular fuzzy number form

\section{CONCLUSIONS}

The aim of this study was to analyse the impact of product life cycle (PLC) on the importance of outsourcing decision-making criteria in a manufacturing firm. As seen, while the investigating activity includes the core competencies of the firm, it is important that they be insourced; but after considering this situation, it is necessary to analyse changes in the importance of other decision-making criteria at each PLC stage. The results of the calculation show that 'the importance of technological capability' and 'the importance of strategic information leakage risk' do not differ across various PLC stages; and it can be assumed that the importance of them is independent of the product life cycle. In contrast, 'the importance of leakage risk of product volume information' and 'the importance of total cost' at different stages of PLC change can be determined by the ordinal logistic regression and estimation of the probabilities for each rating scale. These results create an opportunity for the decision-makers in the case study firm to determine the weights of criteria with respect to the different stages of PLC, and then make decisions on insourcing or outsourcing of manufacturing activities with a suitable method.

\section{REFERENCES}

[1] Aitken, J., Childerhouse, P. \& Towill, D. 2003. The impact of product life cycle on supply chain strategy. Int. J. Production Economics, 85 (2), pp 127-140

[2] Arnold, U. 2000. New dimensions of outsourcing: A combination of transaction cost economics and the core competencies concept. Eur. J. Purchasing Supply Manage, 6, pp 23-29

[3] Bin, G. 2000. Life cycle view of firm's core competence: Chinese manufacturing industry as a case, IEEE, pp 363-367

[4] Birou, L., Fawcett, S.E. \& Magnan, G.M. 1998. Integrating product life cycle and purchasing strategies, International J ournal of Purchasing and Materials Management, 33 (1), pp 23-31

[5] Canez, L., Platt, K.W. \& Probert, D.R. 2000. Developing a framework for make-or-buy decisions, Int. J. Operations Production Management, 20(11), pp 1313-30

[6] Canez, L. 2000. Industrial make-or-buy decisions: Developing a framework and a practical process. PhD thesis, University of Cambridge, Cambridge, United Kingdom.

[7] Chang, S.-L, Wang, R.-C. \& Wang, S.-Y. 2006. Applying fuzzy linguistic quantifier to select supply chain partners at different phases of product life cycle, Int. J. Production Economics, 100, pp 348-359

[8] Che, Z.H. 2009. Pricing strategy and reserved capacity plan based on product life cycle and production function on LCD TV manufacturer, Expert Systems with Applications: An International J ournal, 36 (2), pp 2048-2061 
[9] Chen, J. \& Chen, I. 2010. Using a novel conjunctive MCDM approach based on DEMATEL, fuzzy ANP, and TOPSIS as an innovation support system for Taiwanese higher education, Expert Systems with Applications, 37, pp 1981-1990

[10] Chen, C.-K., Hung, T.-W. \& Weng, T.-C. 2007. Optimal replenishment policies with allowable shortages for a product life cycle, Computers and Mathematics with Applications, 53, pp 15821594

[11] Fill, C. \& Visser, E. 2000. The outsourcing dilemma: A composite approach to the make or buy decision, Management Decision, 38(1), pp 43-50

[12] Fine, C.H. \& Whitney, D.E. 1996. Is the make-or-buy decision process a core competence? MIT Center for Technology, Policy, and Industrial Development, Cambridge, working paper no. 387596.

[13] Van de Henny, W. \& van Peet, H.P. 2006. A decision support model based on the analytic hierarchy process for the make or buy decision in manufacturing. J. Purchasing Supply Manage, 12, pp 258-271

[14] Hoecht, A. \& Trott, P. 2006. Outsourcing, information leakage and the risk of losing technologybased competencies, European Business Review, 18(5), pp 395-412

[15] Hon, K.K.B. \& Xu, S. 2007. Impact of product life cycle on manufacturing systems reconfiguration, Annals of the CIRP, 56, pp 455-458

[16] Hosmer, D.W. \& Lemeshow, S. 2001. Applied logistic regression, J ohn Wiley \& Sons Inc.

[17] Hwang, H.S., Ko, W. \&Goan, M. 2007. Web-based multi-attribute analysis model for make-or-buy decisions, Mathematical Comput. Modeling, 46, pp 1081-1090

[18] J ennings, D. 1997. Strategic guidelines for outsourcing decisions, Strategic Change, 6, pp 85-96

[19] Kajava, J., Anttila, J., Varonen, R., Savola, R. \& Roning, J. 2006. Information security standards and global business, IEEE, pp 2091-2095

[20] Kremic, T., Tukel, O.I. \& Rom, W.0. 2006. Outsourcing decision support: A survey of benefits, risks, and decision factors, Supply Chain Manage. An Int. J., 11(6), pp 467-482

[21] Legendre, P. 2005. Species associations: The Kendall coefficient of concordance revisited, J ournal of Agricultural, Biological and Environmental Statistics, 10, pp 226-245

[22] Ma, K.P. \& Yan, H.S. 2008. Manufacturing mode selection in knowledgeable manufacturing during product life cycle, Int J Adv Manuf Technol., 37, pp 1191-1199

[23] Mantel, S.P., Tatikonda, M.V. \& Liao, Y. 2006. A behavioral study of supply manager decisionmaking: Factors influencing make versus buy evaluation, J. Operations Manag., 24, pp 822-838

[24] Mclvor, R.T., Humphreys, P.K. \& McAleer, W.E. 1997. A strategic model for the formulation of an effective make or buy decision, Manage. Decision, 35(2), pp 169-78

[25] Moses, A. \& Ahlstrom, P. 2008. Dimensions of change in make or buy decision processes, Strategic Outsourcing: An International J ournal, 1(3), pp 230-251

[26] Narasimhan, R., Talluri, S. \& Mahapatra, S.K. 2006. Multi-product, multi-criteria model for supplier selection with product life cycle considerations, Decision Sciences, 37(4), pp 577-603

[27] Ostlin, J., Sundin, E. \& Bjorkman, M. 2009. Product life-cycle implications for remanufacturing strategies, J ournal of Cleaner Production, 17, pp 999-1009

[28] Platts, K.W., Probert, D.R. \& Cáñez, L. 2002. Make vs. buy decisions: A process incorporating multi-attribute decision-making, Int. J. Prod. Econ., 77, pp 247-257

[29] Porter, M. \& Miller, V. 1985. How information gives you competitive advantage, Harvard Business Review, 63(4), pp 149-160

[30] Prahalad, C.K. \& Hamel, G. 1990. The core competence of the corporation, Harvard Business Review, 68(3), pp 79-91

[31] Probert, D.R. 1997. Developing a make-or-buy for manufacturing business. Institute of Electrical Engineers, UK.

[32] Probert, D.R., Cáñez, L.E. \& Platts, K.W. 2001. Technology transfer and succession planning: The relevance of make-or-buy analysis, Proc. Portland Int. Conf. Manage. Eng. Technol., Portland, Oregon, 2, pp 439-443

[33] Reynaldo, J. \& Santos, A. 1999. Cronbach's alpha: A tool for assessing the reliability of scales, J ournal of Extension, 37(2)

[34] Tibben-Lembke, R. 2002. Life after death: Reverse logistics and the product life cycle, Int.J ournal of Physical Distribution \& Logistics Management, 32(3), pp 223-244

[35] Venkatesan, R. 1992. Strategic sourcing: To make or not to make, Harvard Business Review, 70(6), pp 98-107

[36] Wang, J.J. \& Yang, D.L. 2007. Using a hybrid multi-criteria decision aid method for information systems outsourcing, Comput. Operations Res., 34, pp 3691-3700

[37] Welch, J. \& Nayak, A. 1992. Strategic sourcing: a progressive approach to the make-or-buy decision, Acad. Manage. Executive, 6(1), pp 23-31

[38] Wong, H.-K. \& Ellis, P. 2007. Is market orientation affected by the product life cycle? J ournal of World Business, 42, pp 145-156

[39] Zimmermann, H.J . 1987. Fuzzy set, decision making and expert systems, Boston: Kluwer. 